\title{
Planes de gobierno para una sociedad con mayor equidad entre los géneros: una valoración
}

El pasado 20 de septiembre, dentro del Programa de la Mujer de la UCA, se celebró en el auditorio un acto público bajo el título "Propuestas en los planes de gobierno para una sociedad con mayor equidad entre los géneros en orden a profundizar en la democracia". En dicho acto intervinieron los candidatos a la presidencia de los partidos políticos ARENA, Convergencia DemocráticaFMLN y Democracia Cristiana, y a lo largo del mismo, Chávez Mena, Calderón Sol y Zamora Rivas expusieron sus respectivos puntos de vista en torno a la realidad de las mujeres en El Salvador, así como las propuestas de sus programas de gobierno en este aspecto.

Los contenidos de las tres intervenciones, a pesar del inevitable tono electoralista, permiten observar concepciones bien distintas del papel de las mujeres en la sociedad. Igualmente, las diferencias resultan manifiestas en cuanto se refiere a la comprensión y a la sensibilidad ante los fenómenos que atañen a su discriminación, así como a las políticas y voluntades políticas precisas para erradicarla. Pese a todo, e insistiendo en las profundas diferencias apuntadas, de las tres intervenciones puede deducirse un común denominador de generalidad y de inconcreción en las propuestas de gobiemo avanzadas. En este sentido, las mujeres salvadoreñas, a la hora de decidir su voto en unas elecciones que pretenden ser históricas, tendrán que confiar más en la credibilidad genérica que cada partido político les inspire que en los compromisos concretos, públicamente asumidos, en las palabras de los candidatos.

Entrando ya en el análisis de los contenidos de las tres intervenciones, la primera, expuesta por el candidato de ARENA, resultó ser con mucho la más demagógica de todas. Calderón Sol comenzó diciendo que "A pesar del desarrollo alcanzado por la mujer desde fines del siglo pasado, reclamando el derecho al sufragio, aún no hemos reconocido la magnitud de la contribución de la mujer al desarrollo integral de nuestro pueblo ni le hemos dado la plena igualdad". Las causas de esta situación son imputables, desde su punto de vista, a la evolución discriminatoria de la sociedad salvadoreña y a "las condiciones histórico-sociales" que "han determinado una situación diferente para la mujer y el hombre".

Para Calderón Sol, la mujer es víctima de un "rol estereotipado" que le desplaza a un segundo nivel y dificulta su progreso. Este rol, añadió, "comienza en nuestros propios hogares y se extiende a la educación y los lugares de trabajo". Por otra parte, "la paternidad irresponsable, el abandono del hogar y el olvido de los deberes por parte de una gran proporción de los hombres", además de a un heroico y esforzado papel asumido por muchas mujeres, ha dado lugar a una realidad de primer orden: "la mujer ha salido del hogar a trabajar fuera de casa, ha ingresado en la fuerza de trabajo de la nación y por ende se ha convertido en una contribuyente directa al progreso y al desarrollo integral de la sociedad". 
En suma, continuó Calderón Sol, "el estereotipo social de la mujer y la discriminación laboral atentan contra ella", por lo que esta situación debe ser mejorada $y$, especialmente, la de las mujeres que viven sumidas en la pobreza. Con este objeto anunció que su partido está preparando un plan nacional de gobierno que se sustenta en el siguiente principio: "se reconoce a la persona humana como el centro de la actividad de la sociedad y a la familia como el núcleo de la misma". Sobre dicho principio, dijo: "sustentamos la formulación de una política que contempla en toda su magnitud el aporte de la mujer", que debe tender a incrementar las asignaciones presupuestarias en las áreas de salud y educación, porque "si se desea establecer una economía social de mercado... todos los miembros de la sociedad deben tener igualdad de oportunidades para competir".

La segunda parte de su intervención se centró ya concretamente en el "enfoque de la política orientada a la mujer", en donde, Calderón Sol aludió a la Convención sobre la Eliminación de todas las Formas de Discriminación, ratificada por El Salvador, en junio de 1981. Para el Candidato de ARENA, hoy, doce años después de que este tratado internacional pasara a formar parte del ordenamiento interno, se han abierto las oportunidades para la mujer salvadoreña. Desde ahí, y tratando de especificar lo que, con la mejor voluntad, queremos entender como la intención de dar cumplimiento a las obligaciones derivadas de la citada Convención, señaló que "estas oportunidades se orientan en nuestra propuesta de plan de gobierno a políticas específicas en las áreas de legislación, trabajo, educación, igualdad social y acceso a las estructuras de poder y participación de ese 52 por ciento de mujeres que constituyen la población del país. Se suma esto a la protección que se debe dar a la mujer y a la familia, como lo establece el proyecto de ley que preparó la Secretaría Nacional de la Familia y que se encuentra actualmente en la asamblea legislativa".

A continuación, Calderón Sol añadió que en el largo trecho que aún queda por recorrer para lograr la igualdad, "debido a las características machistas de nuestra sociedad, el logro de la igualdad y el reconocimiento del aporte efeclivo de la mu- jer al desarrollo no se logrará sin un esfuerzo conjunto tanto de la sociedad civil como del Estado". Por lo que, en este orden de cosas, a las mujeres les corresponde "organizarse aún más en instituciones voluntarias, tener una participación más activa en política y trabajar para obtener soluciones integrales".

Por su parte, al Estado, "en cumplimiento de su rol subsidiario", le corresponden las siguientes tareas: "estimular la educación de la mujer al igual que la de los hombres, hacer cumplir integralmente las leyes que evitan la discriminación de la mujer, protegerla en sus lugares de trabajo contra el acoso sexual y especialmente de la familia". Finalmente, Calderón Sol terminó su intervención con varias invocaciones a la "mujer-madre", la "pieza más importante de la familia y de la sociedad misma".

A la hora de valorar esta intervención, con toda claridad, saltan a la vista dos aspectos. De un lado, la concepción neoliberal del Estado que preside también su entendimiento de los problemas de las mujeres y de la acción política precisa para abordarlos. De otro, la falta de comprensión del significado real del término discriminación y, por consiguiente, el mantenimiento conceptual de las mujeres en sus papeles más tradicionales y excluyentes.

En cuanto al primero de los aspectos, la formulación última de su discurso queda definida en esta frase: "todos los miembros de la sociedad deben tener igualdad de oportunidades para competir", y en coherencia con ese fin último de competencia, -en definitiva, de mercado-, el papel del Estado, en cuanto a la lucha contra la desigualdad es, como el mismo lo define, "subsidiario". De ahí que ni siquiera se aproxime a definir las políticas específicas en materia legislativa, laboral, educativa, etc., porque desde las concepciones neoliberales cualquier política social vale tanto como su contraria, en la medida en que tienen siempre un carácter fundamentalmente instrumental.

Así, en esta lógica, cuando el Estado interviene estableciendo medidas sociales no persigue otro objetivo que corregir los desequilibrios del merca- 


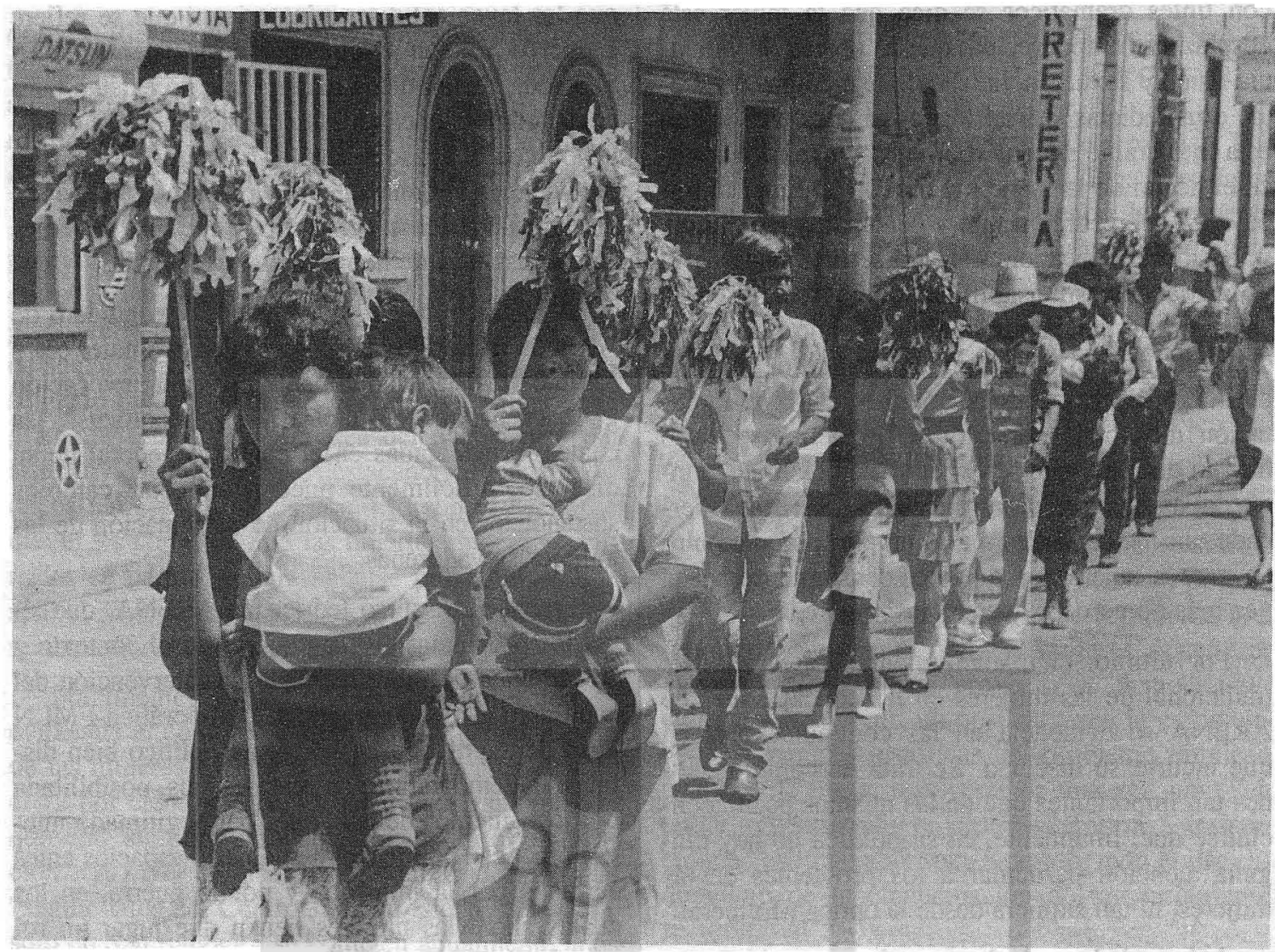

do, y en coherencia con todo lo anterior, cuando Calderón Sol se pone a teorizar sobre las concepciones políticas de su partido repite, como buen neoliberal, que el peso específico de la carga de la lucha por erradicar la desigualdad le corresponde a la sociedad civil, y en el caso de la situación de exclusión de las mujeres, son éstas quienes tienen que "organizarse", "participar" y "buscar soluciones integrales", porque su objetivo político real no es la lucha contra la desigualdad, sino el mejor funcionamiento del mercado.

En este sentido, llama la atención el hecho de que el candidato de ARENA, al comienzo de su intervención, asumiera como principio de su política el reconocimiento de la persona humana como "centro de la actividad de la sociedad". Es esta una formulación sui géneris del artículo 1 de la Constitución, en el que textualmente se dice: "El Salvador reconoce a la persona humana como el origen y fin de la actividad del Estado". Desde esta ćptica constitucional el Estado no puede tener un papel subsidiario en cuanto a los derechos fundamentales de los ciudadanos se refiere, y menos en cuanto a los derechos de los colectivos tradicionalmente excluidos y discriminados como es el caso de las mujeres. El fundamento del Estado es precisamente su garantía, y sin minusvalorar el protagonismo político y social que las organizaciones de mujeres pueden y deben tener en la lucha contra su tradicional discriminación, es al Estado a quien corresponde ejercer una política decidida en su favor, rompiendo incluso las barreras sociales que se enfrentan contra ella.

He aqui entonces las interrogantes que plantea su discurso: mientras se dice que "aún no hemos reconocido la contribución de la mujer al desarrollo integral de nuestro pueblo", no se dice cómo se va producir ese reconocimiento, ni se asumen compromisos de ningún tipo en cuanto a la apertura de espacios para su participación; mientras que 
con tintes dramáticos se dice que la mujer está desplazada, empobrecida, que es objeto de violencia física y sexual, no se dice qué medidas concretas e inmediatas se van a adoptar para acabar con esta intolerable situación; mientras que se reconoce el hecho de la incorporación de la mujer al trabajo, su sobrecarga social y personal como madre, trabajadora, educadora etc., no se dice nada de la obligada corresponsabilidad de los sexos en la asunción de las cargas familiares, sino que se insiste en el papel de las mujeres como el gran eje de la familia y, por consiguiente, centro de imputación último de sus cargas. En definitiva, sólo hay un discurso que se explicita con claridad, aunque ni siquiera se desarrolle: el discurso del mercado, un mercado ahora más amplio porque incluye a esos sujetos productores-consumidores cada vez más numerosos que son las mujeres.

Por último, el mantenimiento del papel más tradicional de las mujeres en las concepciones de ARENA se evidencia en las contradicciones en que incurre su discurso. Se trata de contradicciones tan importantes que de las mismas puede concluirse que, finalmente, en su política no hay ninguna atención particular a los problemas de las mujeres, ni tan siquiera desde la óptica neoliberal.

Asi se deduce de los grandes cometidos que desde su punto de vista tiene que asumir el Estado; en primer lugar del que alude a la educación, planteado por Calderón Sol textualmente en estos términos: "estimular la educación de las mujeres al igual que la de los hombres". Pues bien, si las mujeres están tradicionalmente discriminadas $\mathrm{y}$, en especial, las mujeres pobres, el estímulo de su educación no puede ser igual que el de los hombres, ha de ser distinto, mayor y más intenso, puesto que de lo contrario se estará perpetuando la desigualdad.

Por último, en el marco de esos objetivos aparece también el siguiente: "hacer cumplir integralmente las leyes que evilan la discriminación de la mujer". Tal objetivo no debería ser ni mencionable, pues es una obviedad que a los poderes públicos, y especialmente al poder político, le corresponde cumplir las leyes y velar por el cumplimiento de las mismas. Lo que sucede es que las leyes antidiscriminatorias a que se refiere Calderón Sol son prácticamente inexistentes en El Salvador, de la misma manera que no existen tampoco prohibiciones en las normas laborales en relación con el acoso sexual o, por referimos a algo todavía más vergonzante, la violación sigue siendo en el país un delito perseguible ante los tribunales sólo a instancia de la propia mujer violada.

A lo largo de su intervención, el candidato de ARENA nada dijo que pudiera comprometer estos cambios legislativos, tan solo hizo una invocación genérica a políticas legislativas "especificas", las cuales, consideradas en el contexto general de su discurso, difícilmente pueden anunciar cambios sustanciales en la situación de marginación de las mujeres salvadoreñas.

Frente al discurso teórico de ARENA, que sitúa los problemas de las mujeres en el contexto y en la lógica del libre mercado, la intervención del candidato de Convergencia Democrática-FMLN puso de manifiesto un horizonte político bien distinto: la democratización real del país, posibilitada por los acuerdos de paz, que "han legitimado nuevas prácticas políticas y han abierto espacios antes cerrados por la injusticia y por la guerra, en los cuales ahora las mujeres tienen que jugar un rol decisivo".

Es en este contexto en el que Convergencia Democrática-FMLN entiende la problemática de las mujeres y la necesidad de su resolución, y ello lo entiende, como dijo su candidato, Zamora, como "un imperativo ético y una necesidad histórica", como algo que "es vital para la existencia de una democracia", porque, añadió, "no vamos a tener una sociedad democratizada si mantenemos a las mujeres en la situación de marginación y servidumbre en que históricamente las encontramos". En definitiva, puede decirse que el discurso teórico de esta fuerza política se articulo, en la intervención de su candidato, en torno a la idea central de establecer la real participación de las mujeres en el proceso de democratización del país.

Antes de referirse a las reformas precisas para avanzar en esa democratización, Zamora señaló la necesidad de partir del conocimiento actual de la situación en que se encuentran las mujeres salva- 
doreffas, destacando algunos indicadores suficientemente significativos "que confirman la gravedad del problema". En este sentido, se refirió a siete aspectos de los que indefectiblemente se deduce la condición de exclusión de las mujeres.

Así, aludió en primer lugar al machismo, como práctica social asimilada "por hombres y mujeres de todas las clases sociales y de todas las ideologías... reproducida constantemente por el proceso de socialización desde edades muy tempranas". En segundo lugar se refirió a la clara desventaja de la gran mayoría de las mujeres del país dentro del sistema de salud vigente, destacando algunas realidades tan escalofriantes como lo son el hecho de que el 94 por ciento de las muertes maternas ocuridas en partos pudiera haberse prevenido, o que sólo el 33.6 por ciento de las mujeres embarazadas reciba asistencia prenatal.

Igualmente, señaló que la discriminación se evidencia al comprobar que "sólo el 40 por ciento de las mujeres accede al sistema educativo y sólo el 12 por ciento realiza estudios universitarios, sin que ello signifique su automática profesionalización". A nivel delincuencial, Zamora destacó que "según datos del Centro Judicial Isidro Menéndez, sólo en San Salvador se cometen anualmente unos 12,300 casos de violencia contra mujeres, ...y la mayoría de los casos no llegan a conocerse, pues esta sociedad machista no sólo lo permite, sino que contribuye a la impunidad de ello calificando todos estos delitos como "privados"". La utilización comercial de una imagen de la mujer como objeto de consumo, y la realidad de "una legislación que subordina a la mujer", fueron también aspectos señalados por el candidato de Convergencia Democrática-FMLN, y en este último sentido destacó cómo "la legislación vigente y el sistema judicial siguen permitiendo en la práctica el maltrato y la marginación de las mujeres, a pesar de que el actual gobierno ha firmado tratados internacionales en los que se comprometía a hacer valer sus derechos".

En el plano económico, Zamora puso también de manifiesto la evidente marginación de las mujeres, quienes, "desempeñando un importante rol dentro del sistema productivo... tienen muy limitadas posibilidades de progresar", a pesar de que “constituyen aproximadamente el 40 por ciento de la población económicamente activa registrada, el 53.4 por ciento de nuestro sector informal y son importantes fuentes y receptoras de remesas". Por último, a nivel político, Zamora dijo: "los datos nos señalan claramente que, siendo las mujeres mayoría, ni participan de acuerdo a su número ni están representadas política y socialmente como les corresponde". Y añadió: "es el sistema de división entre los géneros el que ha arrinconado a la mujer a la esfera privada y ha hecho de la esfera pública - donde se ubica la política- un terreno casi exclusivamente masculino".

Tras esta descripción del actual estado de cosas, el candidato de Convergencia DemocráticaFMLN concluyó que, ante la magnitud del problema, las fuerzas políticas a las que representa han asumido plenamente que las mujeres deben ser "una prioridad" en su proyecto de democratización, y tras advertir, no obstante, que la complejidad y las raíces históricas del problema implican que éste no puede resolverse de una vez, sino "en un largo y sostenido proceso de reformas", insistió en que "es imposible seguir postergando el decidido inicio de la emancipación de tan importante sector social".

A partir de ahí y señalando una vez más que en su proyecto político la reivindicación de la situación de la mujer es "un área de acción prioritaria", pasó a establecer las líneas básicas de su propuesta de gobierno que, sin descender al detalle, Zamora fundamentó en "cuatro lineamientos básicos" que pueden resumirse en las siguientes formulaciones: (a) "la política de género debe ser un componente universal de las políticas gubernamentales"; (b) "la acción gubernamental en este campo deberá ser concertadora y concertante"; (c) "la política del gobierno tiene que ser movilizadora y promotora de las condiciones que propicien la ejecución de la reforma. El Estado tiene que ser un instrumento de solución y un posibilitador de los cambios", y (d) "la política de género de nuestro gobierno será participativa".

Finalmente, el candidato de Convergencia Democrática-FMLN terminó con una alentadora proclamación de principios: "el reto para todos es que no seamos neutrales, sino que decididamente nos 
coloquemos en un bando: en el de los oprimidos, de los discriminados, el de las mujeres y desde allí y con toda la sociedad luchemos para que desaparezca la discriminación y la opresión contra la mujer. Cuando lo logremos, entonces seremos neutrales".

Una valoración crítica de esta intervención plantea dos tipos de objeciones. En primer lugar, si la acción política que se pretende seguir ha de ser participativa, "concertada y concertante", parece lógico que las propuestas de gobierno que se presenten sean suficientemente abiertas, dejando espacios para su concreción futura mediante la participación y el consenso de las organizaciones feministas en su determinación última. De ahí que, en principio, no haya nada que objetar, sino todo lo contrario, al compromiso asumido por Zamora cuando afirmó: "nos proponemos desarrollar un esfuerzo concertado con las organizaciones femeninas y organismos afines, para encontrar soluciones progresivas y concretas a esta problemática".

Siendo en principio todo esto razonable, sin embargo, también es cierto que en estos momentos hay ya exigencias muy concretas y muy sentidas, sobre las que existe un sobrado consenso; mas aún, exigencias que se han convertido en auténticos objetivos de principio, compartidos por muchos grupos y organizaciones sociales progresistas y feministas de manera muy generalizada.

En este sentido el candidato de Convergencia Democrática-FMLN se mostró en exceso cauto, pues en cuanto a los cambios precisos de la situación de las mujeres, cuyo carácter injusto y discriminatorio destacó con tanto acierto en la primera parte de su intervención, sin embargo, cuando pasó a especificar las líneas de su política, descendiendo más allá de los cuatro lineamentos generales expuestos, vino a concretar estos aspectos en afirmaciones tan genéricas como las siguientes: "vamos a realizar e impulsar las reformas jurídicas que garanticen la no discriminación de la mujer por razones de sexo o de tipo similar, y la articulación de la mujer a las tareas del desarrollo, en igualdad de condiciones, de oportunidades y de participación"; "habrá que revisar la legislación para garantizar el derecho de la mujer a un trabajo digno" o "vamos a poner paro a esta nociva cultu- ra de la violación sexual".

Por otra parte, siendo lo más positivo de todo su discurso el mensaje inequívoco de participación de las mujeres en todos los aspectos de la vida polílica y social, la visión de esta participación como exigencia de la profundización en la democracia real, e incluso, la formulación general realizada en el sentido de impregnar todas las políticas del Estado de la política de género, hubo un aspecto en la intervención del candidato de Convergencia Democrática-FMLN que cuestiona de manera preocupante la formulación del cuarto de sus lineamientos políticos y, mas aún, puede poner en cuestión también la voluntad decidida que dice tener de no ser neutral en esta lucha, sino de poner sus esfuerzos del lado de las mujeres oprimidas.

Efectivamente, en el cuarto y último lugar de sus lineamientos de acción política en esta materia, Zamora hizo la siguiente afirmación: "la política de género de nuestro gobierno será participativa", y a continuación añadió: "la mayor participación de la mujer en las instituciones y actividades de la nación es fundamental. Hay que implementar mecanismos eficientes que permitan dicha participación, incrementando el número de mujeres en puestos claves del Estado". Sin embargo, dando a entender que no es partidario del sistema de cuotas a la hora del reparto de espacios de poder, fue más allá, añadiendo que "hay que elaborar criterios objetivos y técnicos, que permitan establecer con claridad las capacidades de las personas que van a desempeñar cargos públicos, independientemente de su sexo", y así, "si la aplicación de esos criterios demuestra que una persona es capaz para determinado cargo y esa persona es mujer, no vamos a dudar en nombrarla y en apoyarla en sus tareas".

Sólo faltaría - decimos nosotros - que la coalición de izquierdas más importante en muchos años de historia de este país hiciera lo contrario. No es esta una formulación teórica coherente con la superación de la discriminación y el tradicional apartamiento de las mujeres de la vida política en búsqueda de la promoción de la igualdad: si se rechaza el sistema de cuotas y, efectivamente se quieren establecer criterios objetivos para el desempeño de actividades públicas, pretendiendo a la vez superar esa discriminación y promover la 
igualdad en el acceso de hombres y mujeres a la vida pública, en el actual estado de cosas eso sólo se puede hacer si el sexo es un criterio objetivo más para establecer la adecuación de la persona al cargo. No basta con que a las mujeres no las discriminen en la vida política simplemente por ser mujeres, es que una politica de gobiemo que en verdad quiera promover la igualdad entre los sexos está obligada a establecer "acciones positivas" en su favor, y por consiguiente, a considerar que el hecho de ser mujer es, junto a otros criterios, un valor objetivo de adecuación al cargo público que se va a desempeñar.

Por último, se puede añadir que la prudencia política en el contenido del discurso de Zamora, tal vez comprensible en estos momentos de cam-

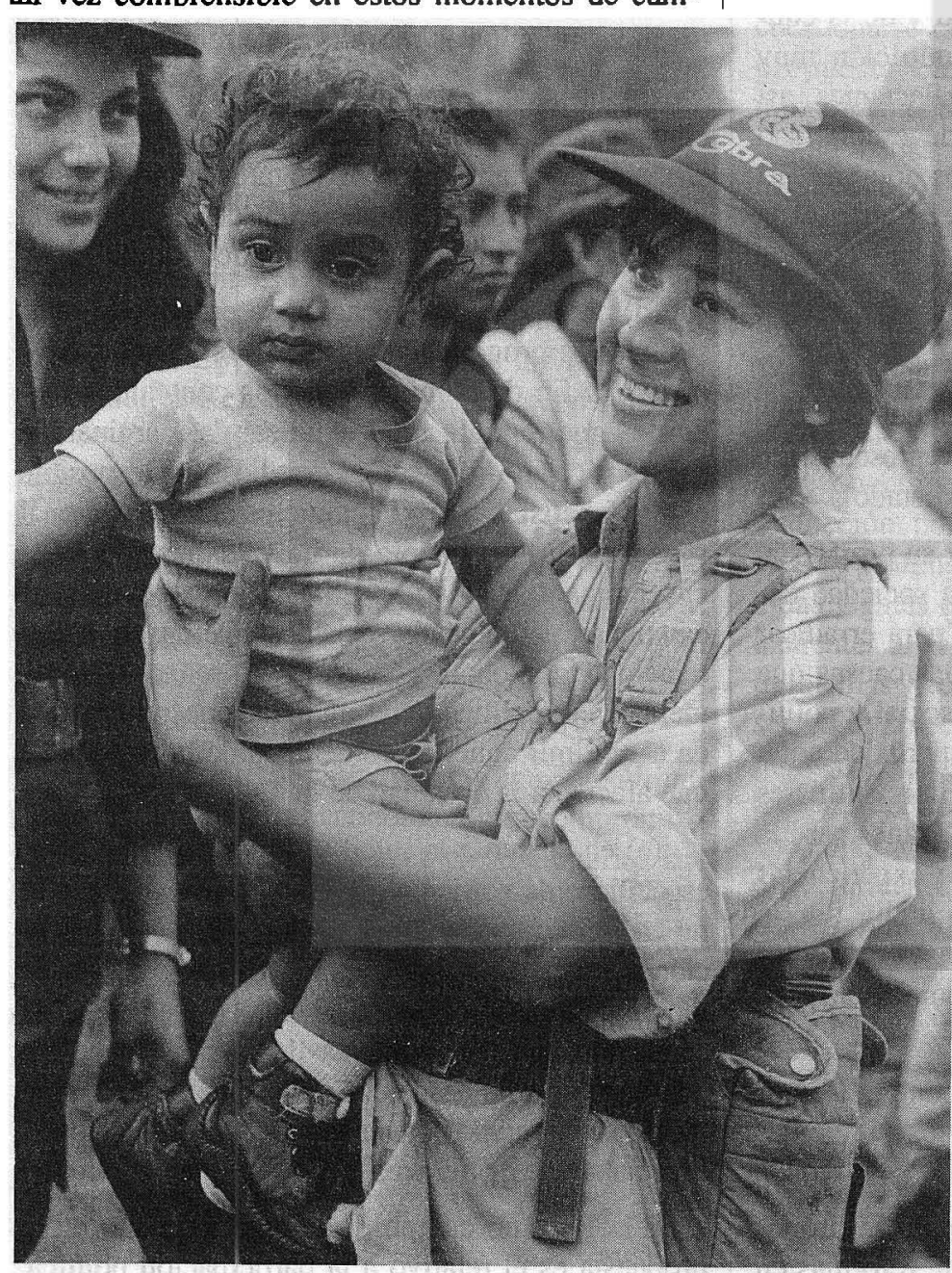

paña y precampaña electoral, llegó demasiado lejos desde el punto de vista del lenguaje. Es significativo observar cómo a lo largo de toda su intervención en ningún momento utilizó el término "feminista" para referirse a las organizaciones de mujeres que reivindican cambios en su situación discriminatoria, tratando de promover la igualdad. El término "feminista", además de contar con una larga tradición histórica - y honrosa en el ámbito de la izquierda - es, por otra parte, el que comúnmente expresa la acción de este tipo de organizaciones. En su lugar, Zamora utilizó el de organizaciones "femeninas", término éste que carece de cualquier tipo connotación beligerante y que más bien viene a servir para referirse a los grupos de mujeres que se reúnen para defender y preservar sus papeles más tradicionales. También es verdad que esta misma crítica se puede hacer al resto de los candidatos, sin embargo, en el ámbito de la izquierda es peligroso asumir con tanta facilidad el carácter "maldito" de algunas palabras y sustituirlas por otras simplemente ambivalentes, porque en el camino se puede perder el valor de progreso que encierran.

La última de las intervenciones correspondió a Chávez Mena, candidato de la Democracia Cristiana quien, omitiendo toda referencia teórica en relación al papel del Estado en la lucha contra la desigualdad de la situación de las mujeres, pasó a formular una propuesta claramente inspirada en los planes de acción contra la discriminación por razón de sexo puestos en práctica en otros países. De ahi que pueda decirse que, a primera vista, los contenidos de su intervención aparecieran como los más concretos y estructurados, pues, como reconoció Chávez Mena, su propuesta se centró en una serie de 
"áreas en las cuales ya existe un nivel de consenso bastante amplio a nivel internacional".

Pero antes de pasar a formularla, al igual que el candidato de Convergencia DemocráticaFMLN, Chávez Mena pasó revista a la situación actual de las mujeres en un análisis que, sin ser tan exhaustivo, vino a coincidir básicamente con la descripción realizada por Zamora, destacando los aspectos más evidentes de la situación de exclusión de la gran mayoría de las mujeres en El Salvador.

Así, se refirió a la desigualdad en las "oportunidades de empleo, de acceso a activos productivos y de ingresos como manifestaciones del papel subordinado de las mujeres en la sociedad". También aludió a la situación de la salud y de la educación, que presentan tasas de desnutrición muy elevadas de mujeres embarazadas y lactantes, así como de analfabetismo y niveles de escolaridad con promedios inferiores a los de los hombres. El maltrato físico, el acoso sexual en el trabajo y la existencia de una legislación obsoleta en lo que se refiere a la familia, el abandono y la paternidad irresponsable, fueron también aspectos destacados y, por último, Chávez Mena hizo mención a los bajos índices de participación polílica de las mujeres que se traducen en una "mínima representación dentro de las instancias de poder del Estado".

Tras este análisis pasó a formular su propuesta, concebida como la exigencia de una sociedad democrática y cristiana, y articulada "para erradicar en el corto, mediano y largo plazo las causas que generan la marginación económica, social y política de las mujeres salvadoreñas". A partir de aquí, la propuesta, elaborada en términos muy similares a los que aparecen en los llamados "Planes para la igualdad de oportunidades", fruto de experiencias europeas y norteamericanas en la lucha por la igualdad, planteó la necesidad de una política "integral" que requiere la actuación combinada y simultánea en distintas áreas.

En síntesis, las acciones que propone la Democracia Cristiana se concentran en los siguientes campos:

(a) en el área de legislación, donde se proponen, también sin excesiva concreción, reformas en el Código de Familia y en la legislación laboral, añadiendo la tarea informativa y difusora de esa nueva legislación mediante el establecimiento de la figura de promotoras y capacitadoras legales. Se propone además que las instituciones vinculadas a derechos humanos establezcan unidades específicas en relación con los derechos de las mujeres;

(b) en el área económica, se plantea una propuesta de fomento productivo, que deberá contener "capacitación y asistencia técnica para productoras del sector informal y microempresas", "acceso legal y real de las mujeres a la propiedad de la tierra, al crédito y a la asistencia técnica", "asistencia financiera" y, promoción del "uso productivo de las remesas entre este sector ya que se ha demostrado que en nuestro país las mujeres constituyen las principales receptoras de remesas";

(c) en materia de política social se plantea "la conveniencia de masificar en las comunidades los sistemas de guarderías y de hogares comunitarios" y se alude al "papel fundamental de la promoción de la organización y capacitación de la mujer";

(d) en el área de educación se propone la revisión de los programas de estudio del sistema educativo "a fin de eliminar aquellos contenidos machistas que reproducen y agudizan la marginación y exclusión de la mujer"; "erradicar en lo posible el analfabetismo entre las mujeres a nivel nacional y garantizar el principio de universalización de la enseñanza básica entre las mujeres"; "diseñar campañas permanentes de capacitación en temas relacionados con el género" y "diseñar e implementar una política de comunicaciones encaminada a suprimir aquellos contenidos de la publicidad que afecten a la dignidad de la mujer";

(e) en cuanto a la salud se proponen programas específicos de nutrición, programas masivos de planificación familiar y "mejorar los sistemas de atención materna a fin de disminuir los altos índices de mortalidad que prevalecen actualmente en el país", añadiendo que "el problema de la salud debe ser abordado no sólo desde el Estado sino desde la misma sociedad civil, potenciando sus organizaciones, especialmente de mujeres";

(e) el último campo de trabajo reseñado en la propuesta es el relativo a la participación política, 
en el que se asume el compromiso de "garantizar la participación de las mujeres dentro de la asamblea legislativa, el poder ejecutivo y la Corte Suprema de Justicia", igualmente se alude a una política de promoción de la organización de las mujeres "en todos los niveles: sindicatos, comunidades, partidos políticos";

(f) por último, se anuncia la creación de un "Consejo Nacional de Mujeres que estará integrado por las organizaciones representativas del movimiento de mujeres, y el cual será una instancia permanente de discusión y elaboración de políticas orientadas a la mujer; al mismo tiempo que tendrá una función de vigilancia en el cumplimiento de los acuerdos y políticas definidos en este organismo de parte de las instituciones encargadas de' su ejecución".

Como fácilmente se puede observar esta es la propuesta más técnica, aunque adolece también de mucha inconcreción en aspectos determinantes como pueden ser los contenidos concretos de las reformas legislativas o los instrumentos especificos para asumir ese "fomento de la producción" del que se habla. En general, resulta bastante positivo el hecho de que para abordar los problemas de las mujeres en El Salvador se busquen soluciones en experiencias y prácticas de gobierno realizadas en países social y económicamente más avanzados, pero estas experiencias demuestran también algo que no se encuentra en la propuesta de Chávez Mena y que consiste en que para llevar a cabo este tipo de planes de igualdad hace falta una decidida voluntad política enunciada en términos de "prioridad" de la acción de gobierno.

Dicha prioridad política significa, en última instancia, la prioridad económica y el compromiso mismo de asignar los recursos presupuestarios suficientes para implementarla. En definitiva, la credibilidad de este tipo de propuestas pasa por una definición expresa en cuanto al incremento claro y decidido de los gastos sociales en el total de los gastos del Estado. Todo este discurso falta en la propuesta de la Democracia Cristiana, y esa ausencia plantea el interrogante del electoralismo. En definitiva, plantea la duda de la verdadera voluntad política de la Democracia Cristiana para que, en su caso, llegado el momento de la acción de gobierno, en verdad vaya a cumplir las promesas formuladas a las mujeres como base del electorado.

En este sentido las dudas se agravan cuando vemos cómo Chávez Mena, en materia de salud, formuló una invocación tan clara al papel de la sociedad civil, puesto que ésta es una área en la que, por principio, el protagonismo le corresponde al Estado y a la que, por consiguiente, hay que destinar muchos recursos económicos. Puede cuestionarse entonces toda la propuesta como una propuesta electoralista $y$, hasta cierto punto, miméticamente reproductora de acciones de gobierno ajenas, puesto que los compromisos económicos que las han hecho posibles en otros lugares no se contemplan en el discurso de este candidato.

En definitiva, como se decía al principio de este análisis, las propuestas de los tres candidatos, aun con sus sustanciales diferencias, dejan muchas interrogantes abiertas y algunos temas importantes por plantear, como por ejemplo, todo lo que atañe al reconocimiento del valor de la diferencia y su traducción política, legislativa, económica y social, la profundización en acciones políticas y medidas legales concebidas como acciones positivas, tendentes al logro de la igualdad, o la discusión de problemas y aspectos tan distintos como pueden ser el establecimiento de cuotas de participación polílica y social o, incluso el problema de la legalización del aborto. En suma, los compromisos en cuanto a medidas concretas que supongan cambios estructurales quedan todavia muy lejos del horizonte político inmediato. 